\title{
The evolution of co-rotating vortices in a canonical boundary layer with inclined jets
}

\author{
Xin Zhang \\ Aeronautics and Astronautics, School of Engineering Sciences, University of Southampton, \\ Southampton SO17 1BJ, United Kingdom
}

(Received 5 May 2003; accepted 9 September 2003; published 24 October 2003)

\begin{abstract}
The evolution of co-rotating streamwise vortices in a canonical flat plate turbulent boundary layer (thickness of the boundary layer $\delta_{0.99}=25 \mathrm{~mm}$ ) is studied. The vortices are produced by an array of inclined jets (diameter $D=14 \mathrm{~mm}$ ) with the same orientation (skew angle $\beta$ and pitch angle $\alpha$ of $45^{\circ}$ ). The focus of the investigation is on the immediate vicinity of the jet exit and downstream locations up to $40 \mathrm{D}$. The Reynolds number based on the diameter of the jet nozzle ranges from 9700 to 29000 , at various jet speed ratios. The main method of investigation is laser Doppler anemometry. Both mean and statistic data are collected and analyzed. The streamwise vortices are a product of complex fluid flow process, featuring horseshoe vortices in front of the nozzle exit, recirculating flow to the lee side of the nozzle, contra-rotating vortices from the rolling up of vortex sheet around the jet, strong and induced spanwise flow. Two types of streamwise vortices are produced: (a) weak vortices at a jet speed ratio $\lambda$ of 0.5 located close to the wall and featuring diametrically opposed, secondary, near-wall flows in between the vortices, (b) strong vortices at higher jet speed ratio featuring significant spanwise movement. The vortices are accompanied by high levels of turbulence, with distinct normal and shear stress distributions. Both turbulence production and convection play important roles in defining the normal stress but only the turbulence production is important in determining the shear stress. (C) 2003 American Institute of Physics.

[DOI: $10.1063 / 1.1624609$ ]
\end{abstract}

\section{INTRODUCTION}

The aim of this work is to examine the near field and the evolution of discrete co-rotating vortices in a canonical flat plate turbulent boundary layer. The vortices are a result of interaction between an array of inclined jets and the boundary layer. Apart from fundamental interests, practical applications could be found in areas such as flow control.

The idea of producing discrete vortices using inclined jets pitched and skewed to freestream was first proposed by Wallis nearly half a century ago, ${ }^{1}$ for the propose of stall control on an aerofoil. The idea was not examined until 1985 when Freestone ${ }^{2}$ conducted some preliminary measurements of surface pressures induced by streamwise vortices. Since then, a number of wind tunnel studies have been performed on different aspects of the flow. Among various workers, Johnston and $\mathrm{Nishi}^{3}$ and Compton and Johnston ${ }^{4}$ conducted detailed surface pressure and pressure probe surveys of single and multiple jet vortex generators, including the effects of the skew angle and velocity ratio. Selby et al. ${ }^{5}$ identified the effects of flow parameters on flow separation over a ramp, using surface pressure tappings and oil flow visualization. McManus et al. ${ }^{6}$ investigated pulsed inclined jets and found them effective in stall control. Zhang ${ }^{7}$ proved that a rectangular nozzle is able to produce a stronger vortex than a circular nozzle with the same mass flow rate, using laser Doppler anemometry (LDA). It is generally recognized that the main advantage of using inclined jets over conventional vanes is a lower and possibly zero drag penalty when not activated, and a potential for active control.
Of the various experimental studies, Selby et $a .^{5}{ }^{5}$ examined the widest range of flow parameters, including jet speed ratio, pitch angle, skew angle, and nozzle diameter. Surface pressure tappings and "oil-dot" flow visualization are used. The work is most useful for engineering design. What is not clear though are the precise physical features associated with the observed surface pressure distributions. Compton and Johnston $^{4}$ are the first to examine the vortex flow field generated by vortex generator jets, using a five hole probe. The work provides the first description of flow physics. However, it is also worth noting that Compton and Johnston have concentrated on the downstream far field locations rather than the jet near field. The first survey position is located at $x / D$ $=20$ and the last one at $x / D=213$, where $x$ is the streamwise distance downstream of the center of nozzle exit. The downstream range, in terms of the ratio between the distance and thickness of the oncoming boundary layer, extends between $x / \delta \simeq 9$ and 97 . The Selby et al. measurements cover up to $x / \delta \simeq 5$. For this type of study it would be useful to examine the jet near field $(x / D \leqslant 10)$, where the flow field still retains the characters of the jet.

The characters of the discrete vortices are influenced by a range of parameters such as skew angle, pitch angle, velocity ratio, size of nozzle, and nozzle orientation. In an earlier work, we reported the development of single and contrarotating vortices. ${ }^{8,9}$ The contra-rotating vortices are able to produce stronger vortices initially. If a pair of inclined jets were used, there would be an upwash region between two main vortices, as against a downwash region produced by 


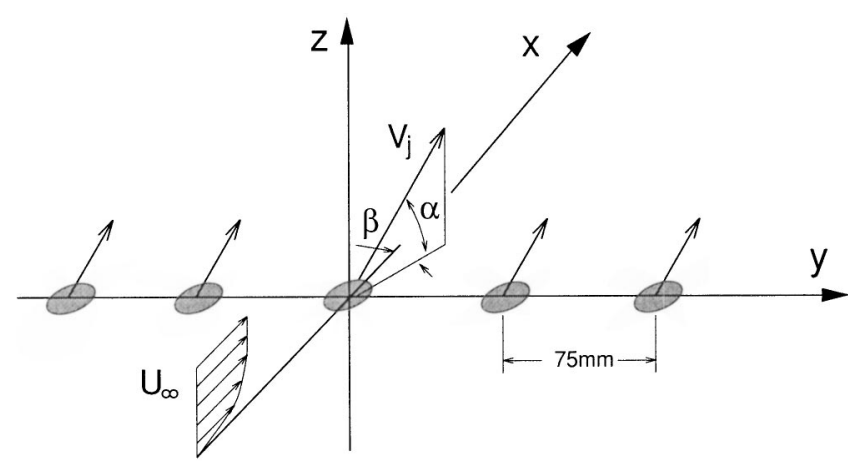

FIG. 1. Schematic of co-rotating, inclined jets.

vortex generator vanes. ${ }^{10}$ The vortices will move away from the wall relatively quickly, in comparison to a single vortex. In the present work, we examine co-rotating vortices produced by an array of inclined jets. One feature of the corotating vortices is their supposed ability to stay closer to an aerodynamic surface. This character could enable them to exercise more effective flow control. This type of flow was studied before, both in model tests ${ }^{4}$ and through numerical modeling. ${ }^{11}$ However, the model tests used pressure tappings and a five hole pressure probe, which do not allow a detailed near field description and turbulence measurements. These are important in providing physical insight and in establishing a numerical model. The numerical modeling effort, though producing good agreement with Compton and Johnston in terms of overall circulation, does not reproduce a number of important flow features, such as the horseshoe vortex and cross flow separation. It is therefore believed that a fresh and in-depth look at the flow field is needed. This study will serve several purposes: (a) to highlight important physical features of the fluid flow, (b) to quantify the downstream evolution of the vortex, and (c) to provide a well validated database in aid of establishing analytical and numerical simulation methods.

\section{DESCRIPTION OF STUDY}

A baseline flow including a canonical flat-plate turbulent boundary layer and inclined jets is selected in the study (Fig. 1). The jets have the same orientation. LDA is used as the main measurement method.

Southampton University's $3.5 \mathrm{~m}$ by $2.6 \mathrm{~m}$ low speed wind tunnel is the test facility. The flat plate boundary layer is established over a $2.43 \mathrm{~m}$ by $2.6 \mathrm{~m}$ by $10 \mathrm{~mm}$ aluminum flat plate placed $0.9 \mathrm{~m}$ above the tunnel floor. A trailing edge flap is employed to establish a zero pressure gradient boundary layer. Tests are conducted at a freestream speed of $20 \mathrm{~m} / \mathrm{s}$ and the freestream turbulence level is $0.3 \%$. Transition is fixed near the leading edge of the plate model. The jets are placed $1.07 \mathrm{~m}$ downstream of the transition position. Figure 2 gives the profile of the boundary layer at $x / D=-7.14$. The fully developed boundary layer has a thickness of $\delta / D$ $=1.786$, and a momentum thickness of $\theta / D=0.196$. Three jet speed ratios, $\lambda$, are used, consistent with earlier studies, at $0.5,1.0$, and 1.5. The jet speed ratio is defined as the ratio between the jet speed, $V_{j}$, and freestream flow speed, $U_{\infty}$.
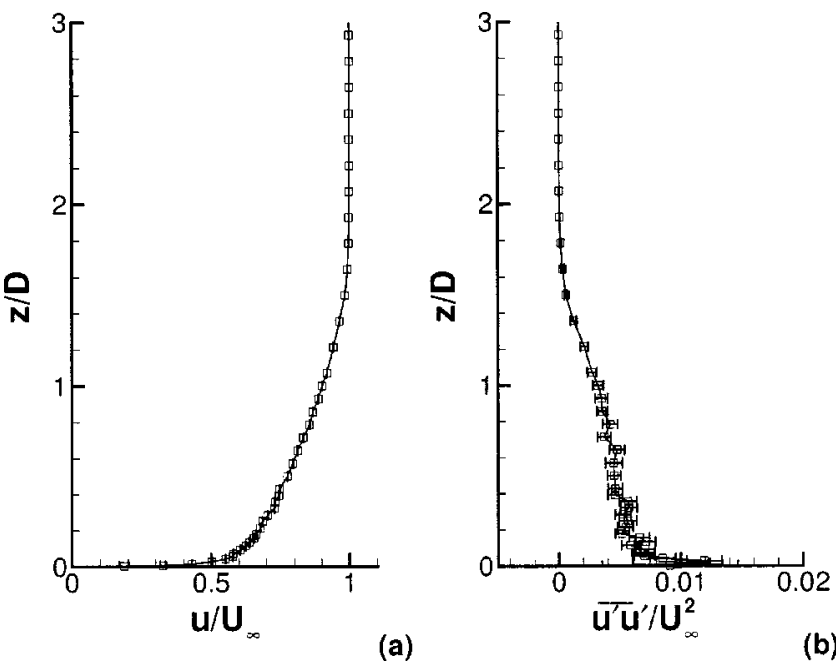

FIG. 2. Oncoming boundary layer at $x / D=-7.14$; (a) $u$ and (b) $\overline{u^{\prime} u^{\prime}}$.

$\operatorname{Re}_{D}$ varies from 9700 to $29000 . \operatorname{Re}_{\theta}$ is 3800 . The value of $\operatorname{Re}_{\theta}$ lies between that of Compton and Johnston ${ }^{4}$ (1500) and Selby et $a .^{5}$ (9000). A total of nine nozzles are used in the tests, with the measurements concentrated on the central nozzle. The nozzles are separated by a spanwise distance of $5.357 \mathrm{D}$. The pitch angle, $\alpha$, and the skew angle, $\beta$, of the jets are both fixed at $45^{\circ}$. The selection follows earlier studies, ${ }^{4,5,12}$ which suggest that the angles are suitable for vortex production.

The jets are generated by a compressed air supply and are regulated twice using a Fisher valve and the control valve on a LDA seed generator. The exit flow is calibrated twice using a pressure probe, before and after each test. The flow proves to be stable over a period of $60 \mathrm{~min}$, which is the time it takes to conduct a LDA survey of 500 positions. Measurements are performed on horizontal planes above the nozzle exit plane and on cross planes between $x / D=5-40$.

A three-component DANTEC laser Doppler anemometer with a $5 \mathrm{~W}$ Ar-ion laser generator is used in the study. The system is operated in an off-axis backscatter mode. Details of the system can be found in Zhang and Collins. ${ }^{12}$ A total of around 500 positions are surveyed during each test, with 1500-2500 samples collected for each position. Natural cubic splines are used to obtain the derivatives of $u, v, w$ with respect to the measurement coordinates $x, y, z$. It should be noted that the coordinate system used in presenting the results is a left-hand system (see Fig. 1). This arrangement follows the wind tunnel coordinate system. The flow outside the jet is seeded using a water based seeding device (JEM Hydrosonic 2000). The seedings are not introduced in front of the test model, which would affect accuracy of the measurements. Rather, a venting system located behind the tunnel working section and in front of the fan rotor is used to duct the seedings into the tunnel through the tunnel refresh vents.

The uncertainties and 95\% confidence interval in the LDA data are estimated using procedures given by Moffat ${ }^{13}$ and Benedict and Gould, ${ }^{14}$ respectively. The uncertainties in the $x, y, z$ position measurements are $\pm 0.16 \mathrm{~mm}$. Uncertain- 


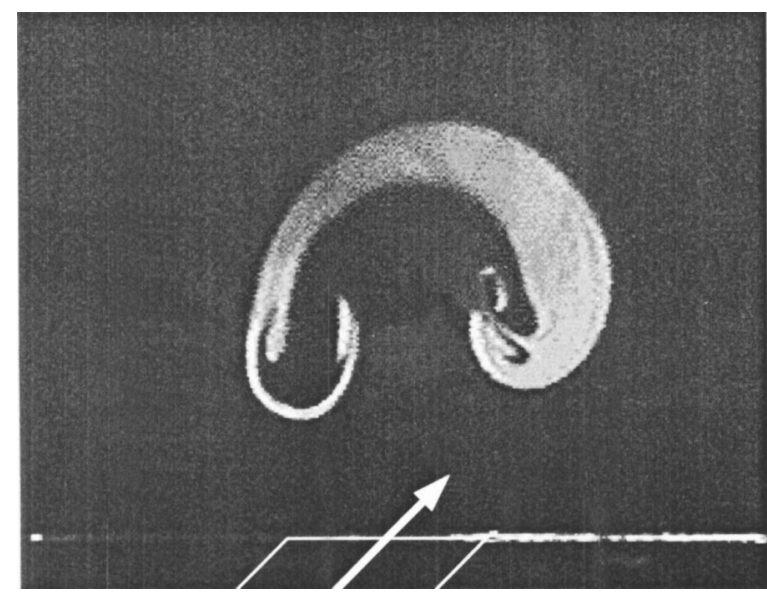

FIG. 3. Laser light sheet visualization showing the formation of streamwise vortices at $\lambda=1.0 ; \operatorname{Re}_{D}=1940, x / D=3.0$. The jet is seeded. View from upstream.

ties in the pitch and skew angles of the jet are $\pm 0.25^{\circ}$. The tunnel speed is maintained to an accuracy of $\pm 0.05 \mathrm{~m} / \mathrm{s}$. The uncertainties in the velocity measurements are $\pm 0.1, \pm 0.3$, and $\pm 0.1 \mathrm{~m} / \mathrm{s}$ for the three velocity components, $u, v, w$. The $95 \%$ confidence intervals for the turbulence stress terms are: $\overline{u u}, \overline{w w}< \pm 14 \% ; \overline{v v}, \overline{u v}, \overline{v w}< \pm 17 \%$; and $\overline{u w}< \pm 14 \%$. Finally it should be mentioned that in calculating the circulation level, the non-dimensional vorticity data with $|\Omega|$ $<0.02$ are ignored. This practice ensures that the "noise" in the measured data will not mask the overall circulation.

\section{RESULTS AND DISCUSSIONS}

\section{A. Initial vortex generation}

When an inclined jet is introduced into a cross flow, streamwise vortices are formed, which evolve downstream. If the jet is not skewed, a pair of contra-rotating vortices will ensue. It is generally acknowledged that the initial phase of streamwise vortex formation could be modeled as an inviscid process. The first attempt at studying the vortex formation process was due to Chen, ${ }^{15}$ looking at a circular jet exhausting normally from a flat plate into a uniform cross-stream. The jet surface is usually treated as a vortex sheet represented by a system of longitudinal and ring vortex filaments. The longitudinal vortex filaments are due to the cross flow and the ring vortex filaments to the jet flow. The strength of the vortex sheet can be determined by the potential flow theory. ${ }^{16}$ The interaction between the oncoming flow and the vortex sheet leads to the rolling-up of the longitudinal vortex filaments to form two large vortices in the jet direction. After the jet eventually bends toward the cross flow, the vortices become the so-called streamwise vortices.

If the jet carries a skewed angle in addition to a pitched angle, a spanwise velocity component is introduced. As a result, one of the vortices in the vortex pair is enhanced and the other weakened. This process is best illustrated in Fig. 3. Figure 3 is obtained by laser light sheet visualization at a lower $\operatorname{Re}_{D}$ than the actual model test $\operatorname{Re}_{D}$ values. It enables the vortex formation process to be viewed clearly and in the meantime retains the main physical features. The core of the jet is highlighted by the bright region which represents the seeded core of the jet. At a distance of $x / D=3$, it assumes a kidney shape as a result of the rolling up of the longitudinal vortex filaments. The left-side vortex is now the dominant one, which is enhanced by the induced spanwise flow due to the skewed jet. It will continue downstream as this mechanism remains. The right-hand vortex is the weaker one. It can be surmised that, if the core of the jet is turbulent and the skew angle of the jet is large, the right-hand vortex will quickly be diffused, leaving a dominant left-hand vortex. It is this vortex which is commonly referred to as the flow control vortex. On the other hand, it can be expected that, if the spanwise velocity component is small, the pair of vortices will be able to persist downstream over a large distance.

\section{B. Jet near field}

A number of physical features accompany the formation of the vortices. These include horseshoe vortices, recirculating regions, induced spanwise flow, etc. The complex nature of the near field flow is illustrated by the surface streakline patterns shown in Fig. 4. Figure 4 gives two representative surface flow patterns, which are associated with two types of vortex production (see also section Sec. III C). The surface flow at $\lambda=1.5$ is rather similar to the one at $\lambda=1.0$; it is therefore not included in Fig. 4. The major features are highlighted for the $\lambda=1.0$ flow. These features are separated by "critical points" such as saddle points and "critical lines" such as bifurcation lines.

We now inspect the $\lambda=1.0$ case. In front of the jet exit, there is a horseshoe vortex formed by the separated flow immediately in front of the jet. This horseshoe vortex wraps around the jet exit and extends downstream. It is heavily skewed toward the jet exit direction. The horseshoe vortex is marked by a bifurcation line and a saddle point, which separate the oncoming flow from the horseshoe vortex. Behind the jet exit, the cross flow around the jet separates on the lee side of the exit. The two separation regions are indicated on the oil flow by the two pools of unevaporated oil residue. The skewed jet is seen to induce strong spanwise cross flow behind the jet exit. The main induced surface flow is separated from that due to the secondary flow from the other side by a bifurcation line, which extends downstream. It is clear that the induced spanwise surface flow due to the secondary flow from the skew side quickly disappears and the spanwise movement of the surface flow after $x / D \approx 10$ is then dominated by the main streamwise vortex. The induced spanwise flow by the skewed jet and that by the main streamwise vortex merges seamlessly downstream on the surface flow streakline patterns.

For the $\lambda=0.5$ flow, the surface flow pattern reveals some different features from that at $\lambda=1.0$. There does not seem to be a horseshoe vortex in front of the jet exit. The spanwise movement of the surface flow behind the jet exit is less pronounced than that at $\lambda=1.0$, which is an indication of weak streamwise vortices. In fact, the surface streaklines suggest that after $x / D \approx 10$ there is little discernible spanwise movement at this jet speed ratio. This is an interesting but unexpected feature (see additional discussions in Sec. III C). 


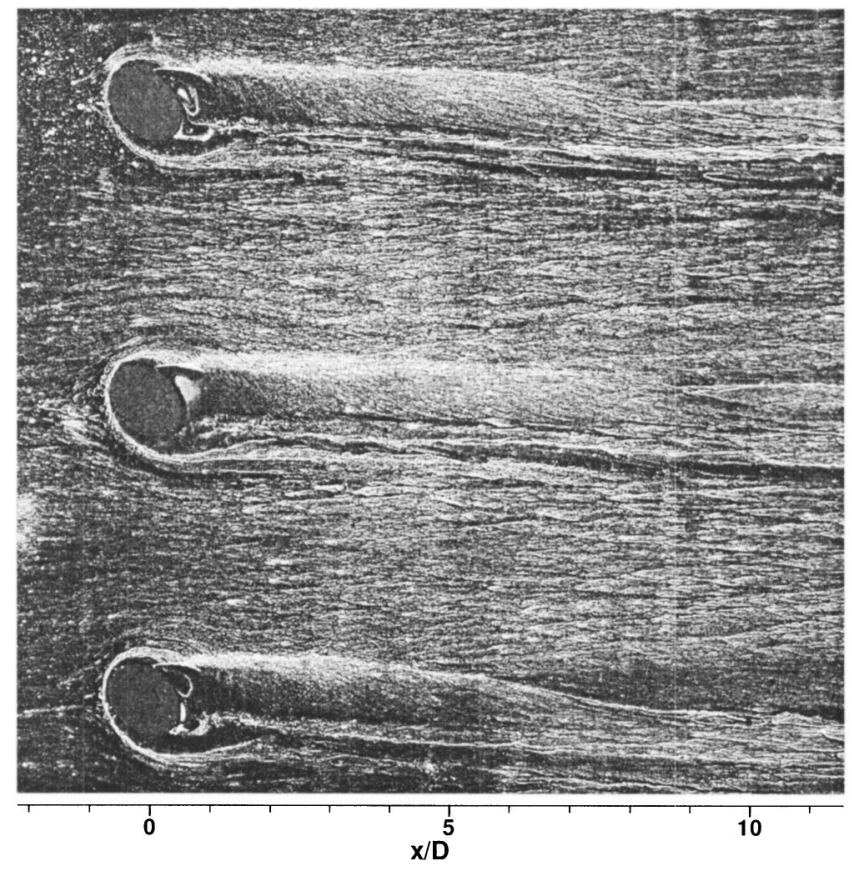

(a) Formation of a weak vortex; $\lambda=0.5$.

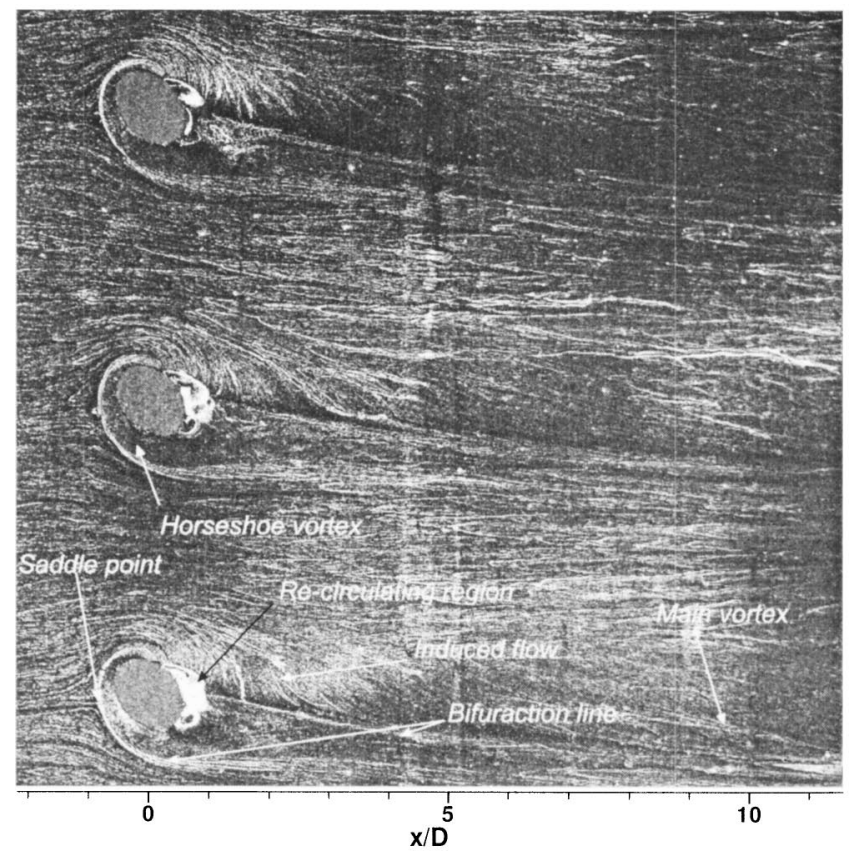

(b) Formation of a strong vortex; $\lambda=1.0$.

FIG. 4. Oil flow visualization. Flow from left to right

A bifurcation line exists downstream of the jet exit, separating the induced flows by two streamwise vortices. The recirculating regions behind the jet exit still exist.

To further quantify the near wall flow around the jet exit, a detailed survey was conducted at $z / D=0.125$ above the wall for the $\lambda=1.0$ jet. Figure 5 gives the velocity vectors and sectional streamlines. It should be noted that the sectional streamlines can be misleading as the measurement plane does contain significant cross plane flow. They should therefore be viewed with caution. They do, however, reveal some interesting features of the near wall flow. It is immedi-
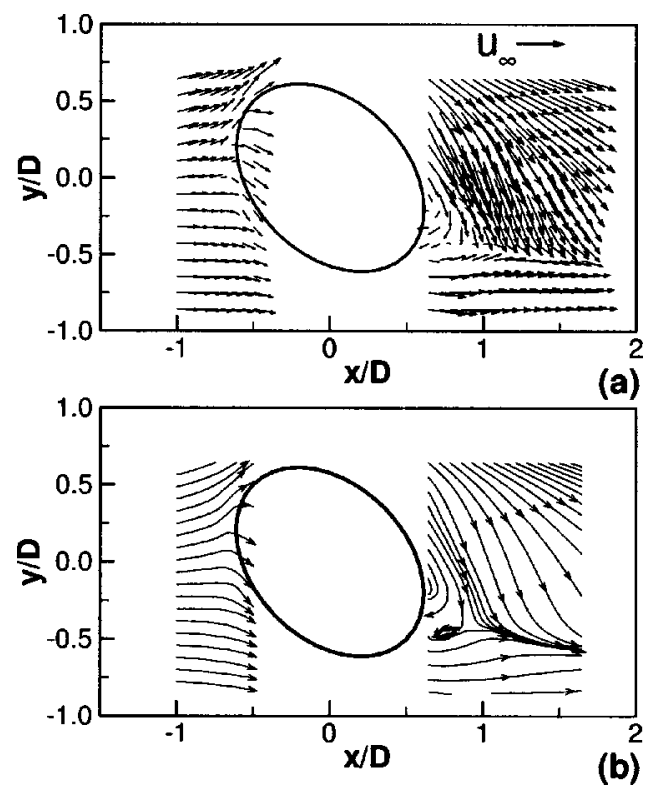

FIG. 5. Flow pattern near the jet exit at $\lambda=1.0, z / D=0.125$; (a) velocity vectors, (b) sectional streamlines.

ately obvious that (a) there is a recirculating flow region behind the jet on the horizontal plane and (b) the horseshoe vortex as observed in the oil flow (Fig. 4) does not have a large transverse extent. Behind the jet, the sectional streamlines features an asymptotic line which is located further away in spanwise extent from the bifurcation line on the wall (see Fig. 4). This feature suggests that the flow changes significantly in the transverse direction immediately above the wall. Above the asymptotic line in Fig. 5(b), we note the presence of a strong cross flow. The strong near wall cross flow helps to generate one of the streamwise vortices and quickly establishes it as the dominant one.

Due to seeding problems, the measurements at $z / D$ $=0.125$ do not extend to the region occupied by the jet. Many detailed near wall measurements are conducted at a larger distance of $z / D=0.25$. Figure 6 confirms the existence of strong spanwise flow at $\lambda=1.0$, which is induced by the flow around the skewed jet. It should be considered as a contributing factor in generating the dominant vortex. The $T K E$ contours in Fig. 7 are plotted at the same intervals for the three cases. They reveal that a highly turbulent region exists to the lee side of the jet exit, corresponding to the separated flow regions. Although the jet still possesses a potential core, high level turbulence stress is generated through the jet and cross flow interaction, which marks the boundary between the jet and the cross flow. This feature is much more obvious at higher jet speed ratios.

\section{Mean vortex flow}

The evolution of the streamwise vortices is examined in a series of measurements at various cross planes ranging from $x / D=5$ to 40 . Examples of the streamwise vortices and their movement are given in Figs. 8 and 9. The vortices at $\lambda=1.0$ have the same characters as those at $\lambda=1.5$ and are not included. A summary of the main parameters of the dis- 


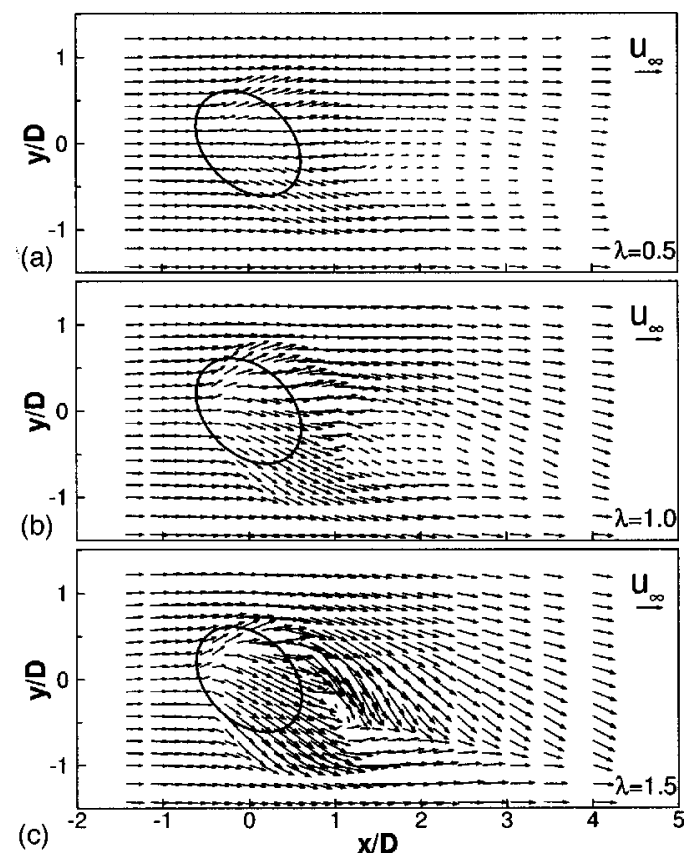

FIG. 6. Velocity vectors above the jet exit at $z / D=0.25$; (a) $\lambda=0.5$, (b) $\lambda=1.0$, and (c) $\lambda=1.5$.

crete vortex is given in Table I. The vortices are seen to move further in the spanwise direction than that produced by a single nozzle. For example, at $\lambda=1.0$ and $x / D=5$, the current arrangement gives the center of vortex location at $\left(y_{c} / D=0.538, z_{c} / D=0.704\right)$, while single jet measurements give the center of vortex location at $\left(y_{c} / D=0.244\right.$, $z_{c} / D=0.732$ ). At $\lambda=0.5$ and $x / D=5$, the center of vortex is located at $\left(y_{c} / D=0.158, z_{c} / D=0.599\right)$ for the co-rotating jets and $\left(y_{c} / D=-0.039, z_{c} / D=0.462\right)$ for the single jet. At

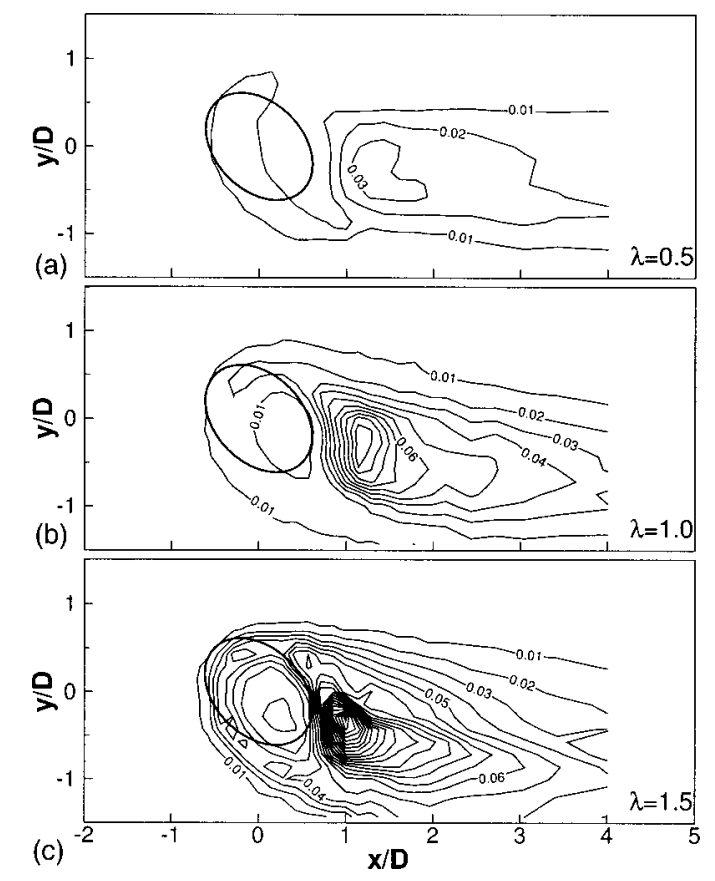

FIG. 7. TKE contours above the jet exit at $z / D=0.25$; (a) $\lambda=0.5$, (b) $\lambda=1.0$, and (c) $\lambda=1.5$.
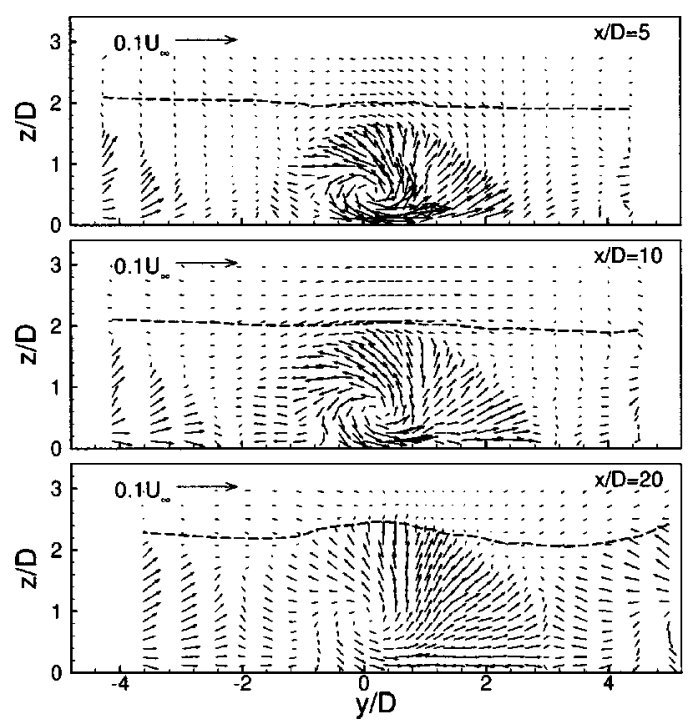

FIG. 8. Vortex development at $\lambda=0.5$; velocity vectors. Dashed line: edge of boundary layer at $\delta_{0.99}$.

$\lambda=1.5$ and $x / D=5$, the center of vortex is located at $\left(y_{c} / D=0.536, z_{c} / D=0.973\right)$ for the co-rotating jets and $\left(y_{c} / D=0.252, z_{c} / D=1.154\right)$ for the single jet. The difference in the transverse movement though is not large.
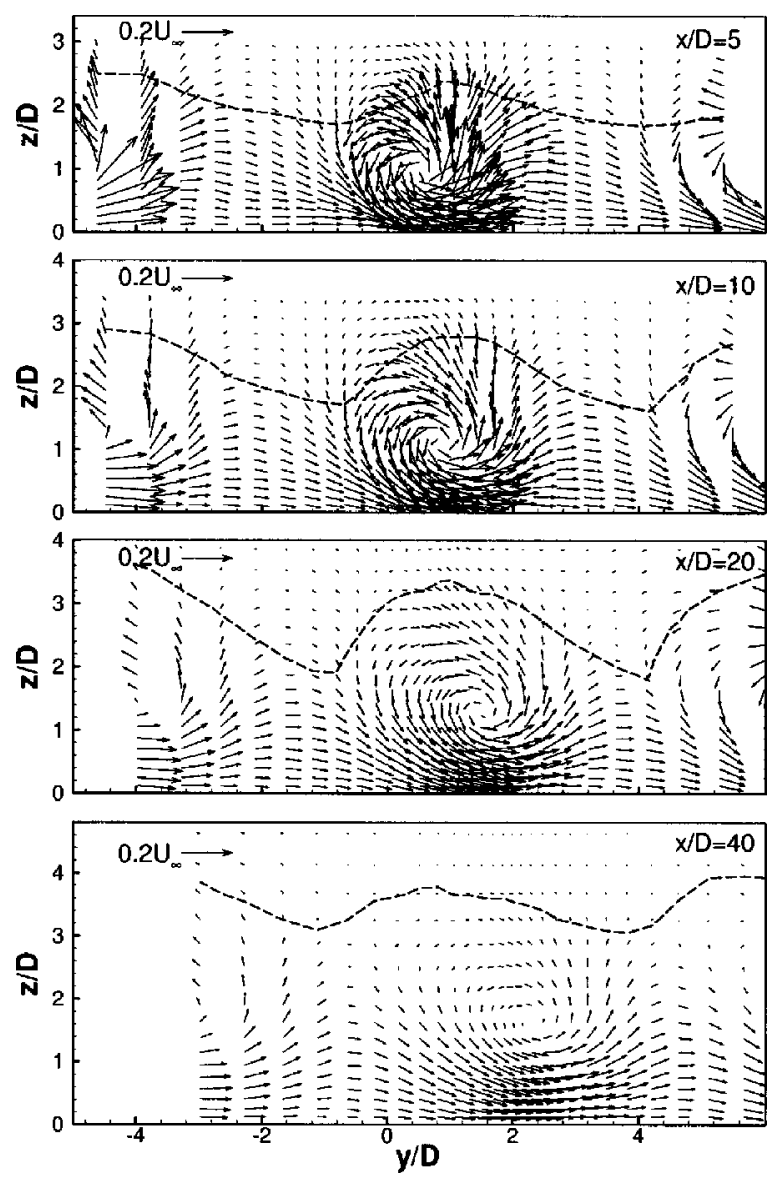

FIG. 9. Vortex development at $\lambda=1.5$; velocity vectors. Dashed line: edge of boundary layer at $\delta_{0.99}$. 
TABLE I. Position and strength of vortex. Subscript $c$ refers to the center of vortex and $m$ the maximum vorticity. $\Omega$ is the normalized streamwise vorticity, $(\partial w / \partial y-\partial v / \partial z) D / U_{\infty} . \Gamma$ is the circulation level, normalized by $U_{\infty} D$.

\begin{tabular}{|c|c|c|c|c|c|c|c|c|c|c|c|c|c|}
\hline \multirow[b]{2}{*}{$x / \mathrm{D}$} & \multicolumn{3}{|c|}{$\lambda=0.5$} & \multicolumn{5}{|c|}{$\lambda=1.0$} & \multicolumn{5}{|c|}{$\lambda=1.5$} \\
\hline & 5 & 10 & 20 & 5 & 10 & 20 & 30 & 40 & 5 & 10 & 20 & 30 & 40 \\
\hline$\Omega_{c}$ & -0.300 & -0.171 & -0.071 & -0.681 & -0.361 & -0.185 & -0.140 & -0.075 & -1.404 & -0.752 & -0.377 & -0.234 & -0.156 \\
\hline$y_{c} / D$ & 0.158 & 0.241 & 0.002 & 0.538 & 0.765 & 1.392 & 1.606 & 1.668 & 0.536 & 0.867 & 1.490 & 1.930 & 2.300 \\
\hline$z_{c} / D$ & 0.599 & 0.621 & 0.870 & 0.704 & 0.808 & 1.014 & 1.221 & 1.389 & 0.973 & 1.100 & 1.300 & 1.490 & 1.640 \\
\hline$\Omega_{m}$ & -0.515 & -0.213 & -0.079 & -1.075 & -0.547 & -0.265 & -0.200 & -0.144 & -1.610 & -0.843 & -0.480 & -0.330 & -0.224 \\
\hline$y_{m} / D$ & 0.357 & 0.446 & 0.092 & 0.660 & 0.982 & 1.487 & 1.750 & 1.982 & 0.661 & 0.987 & 1.487 & 1.987 & 2.528 \\
\hline$z_{m} / D$ & 0.250 & 0.191 & 0.829 & 0.523 & 0.531 & 0.699 & 0.725 & 0.905 & 0.809 & 1.002 & 1.049 & 1.098 & 1.058 \\
\hline$\Gamma$ & -0.154 & -0.119 & -0.029 & -0.571 & -0.461 & -0.346 & -0.296 & -0.254 & -0.813 & -0.663 & -0.573 & -0.501 & -0.450 \\
\hline
\end{tabular}

It is immediately clear that there exist two types of vortex: a strong vortex at higher jet speed ratio $(\lambda=1.0$ and 1.5) and a weak vortex at $\lambda=0.5$. The strong vortex moves in both spanwise $(y)$ and transverse $(z)$ directions in a monotonic fashion. It moves away from the wall as it evolves downstream. Measurements of the primary velocity in the streamwise direction show that the boundary layer experiences large changes. The weak vortex at $\lambda=0.5$ induces relative small changes to the primary velocity field, as illustrated by the contour of the edge of the boundary layer in Fig. 8. The vortices experience smaller movement in the spanwise direction than those at $\lambda=1.0$ and 1.5 and are deeply embedded inside the boundary layer. For both types of vortices, surface flow visualization and velocity survey show no sign of induced cross flow separation. Indeed it should be noted that the type of streamwise vortex reported here is different from those generated through separation off an edge on an aerodynamic surface. ${ }^{17}$ The strength of the vortex is an order of magnitude lower than that reported by Cutler and Bradshaw.

A major difference between the results at $\lambda=0.5$ and those at $\lambda=1.0$ and 1.5 does lie in the induced secondary flow between the adjacent co-rotating vortices distributed in the spanwise direction. At $\lambda=1.0$ and 1.5 , the near wall flow beneath the center of vortex moves in the same direction in the cross plane in the direction of the skew. This feature can be seen clearly in Fig. 9. The cross plane flow is particularly strong just beneath the center of vortex. Although turbulent diffusion increases the size and reduces the strength of the vortices, the vortices do retain their distinct characters until $x / D=40$, which is the last survey station. At $\lambda=0.5$, the secondary cross flow between the adjacent co-rotating vortices displays a different character. The secondary flows induced by the adjacent vortices are diametrically opposite to each other in the area between the vortices. This feature is clearly seen in Fig. 8. It reflects the influence of the jet, which in this case exerts a bigger impact on the induced cross flow than that at higher jet speed ratios. Although a first look at Fig. 8 shows the circulating flow due to the main streamwise vortex, a concentration of secondary vorticity does exist to the left of each main vortex. This feature explains the existence of the diametrically opposite secondary flows between the adjacent vortices.

To highlight the difference between the two types of vortex distributions, we plot the primary velocity distribution along a line between the center of vortex and the wall, at $z / D=0.25$. Results are given in Fig. 10. Two other velocity components $(v, w)$ would give the same information about the flow. The particular feature to examine is the relative positions of the peak and trough (dip) in the distribution which reflect the strength and position of the vortex and the jet. At $\lambda=1.0$ and 1.5 , the influence of the main vortex is clearly seen. At $x / D \geqslant 10$, the main vortex induces a peak and a dip in the primary velocity distribution [Figs. 10(b) and 10(c)]. These move in the spanwise direction as the vortices evolve downstream. However, in the jet near field, an addi-
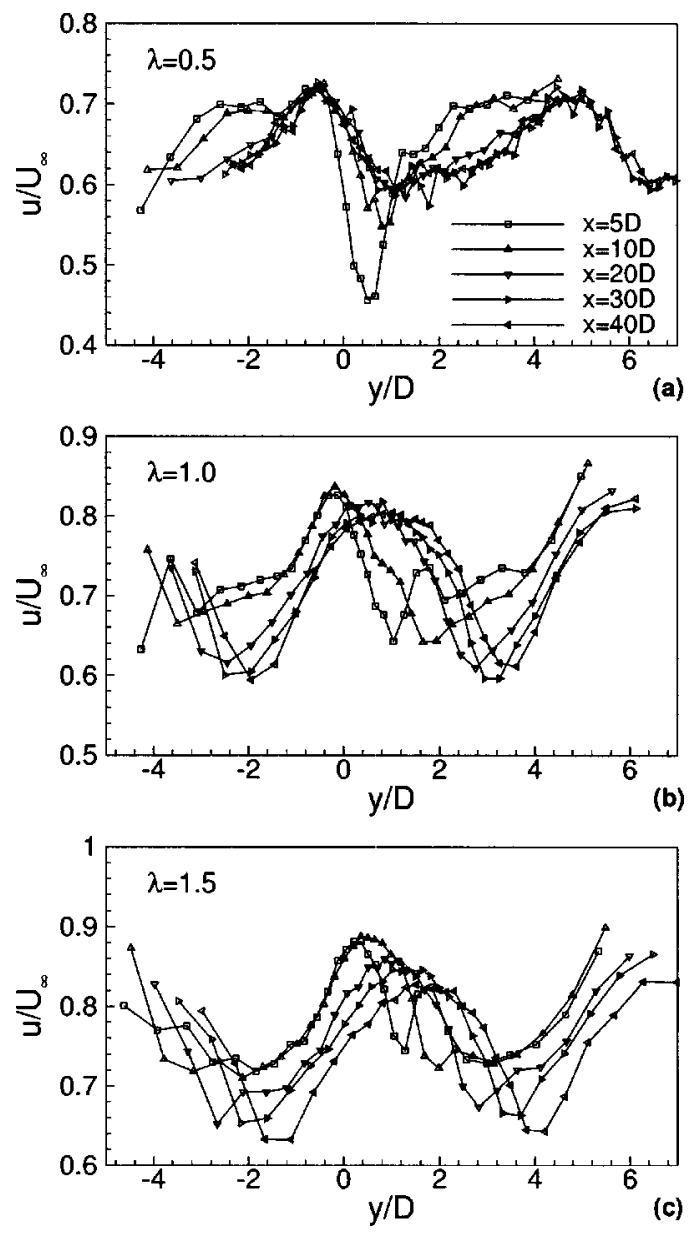

FIG. 10. Streamwise velocity at $z / D=0.25$; (a) $\lambda=0.5$, (b) $\lambda=1.0$, and (c) $\lambda=1.5$. 
tional peak in the primary velocity appears to the left of the dip. At $\lambda=1.0$ and $x / D=5$, the dip is at $y / D=1.02$, the main peak to the left at $y / D=-0.09$, and the additional peak to the right of the dip at $y / D=1.79$. At $x / D=10$, the dip moves to the right at $y / D=1.68$. However the main peak stays at the same position at $y / D=-0.09$. The relative difference between the main peak and the dip stays the same between $x / D=5$ and 10 . There is no secondary peak to the right of the dip at $x / D=10$, an indication of the diminishing effect of the jet. At $\lambda=1.5$ and $x / D=5$, the dip is at $y / D=1.29$, the main peak to the left at $y / D=0.37$, and the additional peak to the right of the dip at $y / D=1.79$. At $x / D=10$ the main peak stays at the same position but becomes much broader. The dip is located at $y / D=1.98$. A rather small but discernible second peak is now at $y / D=2.32$.

At $\lambda=0.5$, a dip in the primary velocity exists at $y / D$ $=0.49$. The primary velocity rises to either side of the dip position. To the right of the dip, there is a peak position at $y / D=-0.51$. This type of distribution is typical of that due to an inclined jet. Further downstream at $x / D=10$, a dip still exits at $y / D=0.87$. The peak to the left experiences little movement. The relative difference in the primary velocity between the dip and the peak on the right has reduced significantly. Between $x / D=10$ and 20 , there is only a small change in the distribution. However, beyond $x / D=20$ there is little change in the induced primary distribution, both in position and level. The results also confirm the earlier observation of position using oil flow visualization. This is both interesting and unexpected as one would expect an array of streamwise vortices to move in the spanwise direction, under the influence of the wall. However, as we noted before this particular arrangement enables the jet to produce diametrically opposite secondary flows between the adjacent vortices. The effect is to counter the effect of the wall. It seems that a judicious use of the jets and vortex properties could lead to deeply embedded co-rotating vortices in a turbulent boundary layer, with an ability to retain induced velocity strength over a large distance. The flow physics reported here could be used to design effective jet vortex generators. One advantage lies in the low jet speed ratio (below unity), which would enable ram air to be used without the need of an expensive air supply.

\section{Turbulence stresses}

The evolution of vortex is influenced by the turbulence stresses in the boundary layer. We now examine the normal stress, $\overline{u^{\prime} u^{\prime}}$ and the primary shear stress, $-\overline{u^{\prime} w^{\prime}}$. Figures 11 and 12 give examples of typical $\overline{u^{\prime} u^{\prime}}$ distributions and Figs. 13 and 14 the $-\overline{u^{\prime} w^{\prime}}$ distributions. Again the $\lambda=1.0$ results are not shown as they are similar to the $\lambda=1.5$ ones. The center of vortex and the position of the maximum vorticity are also added to the figures. The maximum vorticity occurs below and to the upwash (right) side of the center of vortex, which is a well-known feature for this type of flow.

The $\overline{u^{\prime} u^{\prime}}$ distributions show the influence of the jet and high level normal stresses in the near field of the jet. At $x / D=5$ and $\lambda=0.5$ (Fig. 11), the influence of the jet is clear. Closed contour lines above the center of vortex assume a
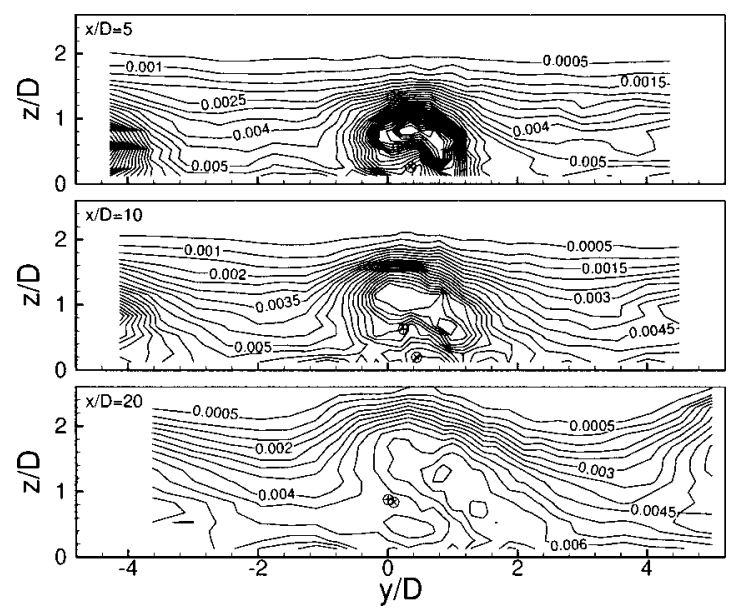

FIG. 11. Vortex-development at $\lambda=0.5$; normal stress $\overline{u^{\prime} u^{\prime}} / U_{\infty}^{2}$. $(\oplus)$ Center of vortex and $(\otimes)$ maximum vorticity.

kidney shape and mark the high level concentration of the normal stress. This feature is retained at $x / D=10$. The effect of the main vortex begins to manifest itself at $x / D=20$. At this streamwise location, the vortex brings a concentration of low $\overline{u^{\prime} u^{\prime}}$ distribution to the near wall region just below the center of vortex and there is a high level concentration to the upwash side of the vortex. At $\lambda=1.5$, the presence of the jet is quite pronounced at $x / D=5$ and 10 (Fig. 12). High levels of $\overline{u^{\prime} u^{\prime}}$ distribution could be found above the center of vortex. At $x / D=5$, the closed contour lines also have a kidney shape, surrounded by closely packed contour lines. The influence of the jet is also marked by the closed contour lines which are not found after $x / D=20$. The main vortex begins to exert bigger influence on the normal stress distributions as
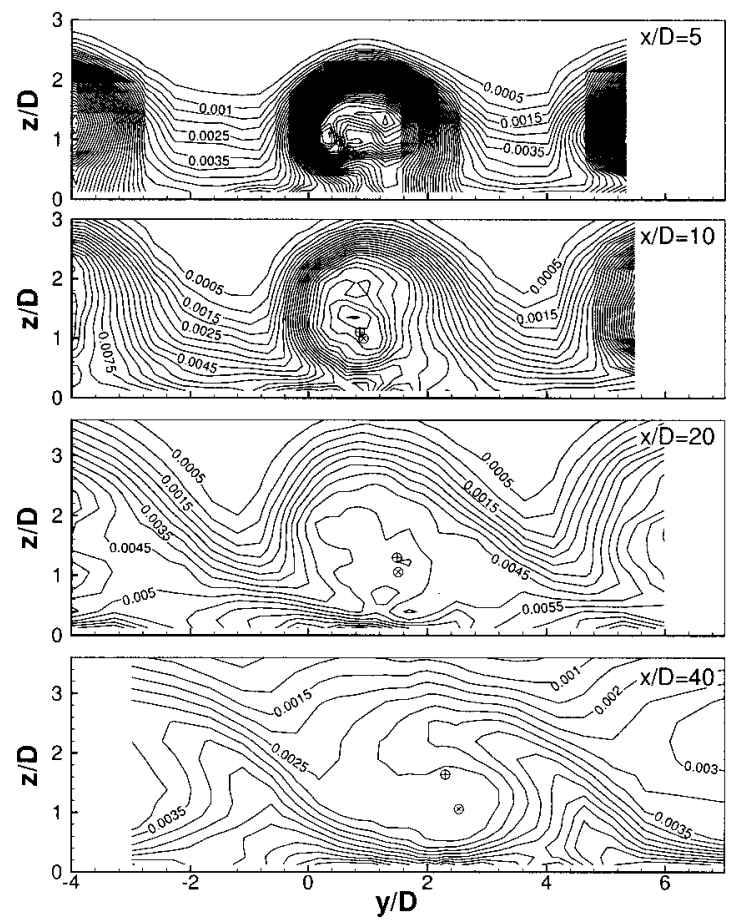

FIG. 12. Vortex development at $\lambda=1.5$; normal stress $\overline{u^{\prime} u^{\prime}} / U_{\infty}^{2}$. ( $\oplus$ ) Center of vortex and $(\otimes)$ maximum vorticity. 

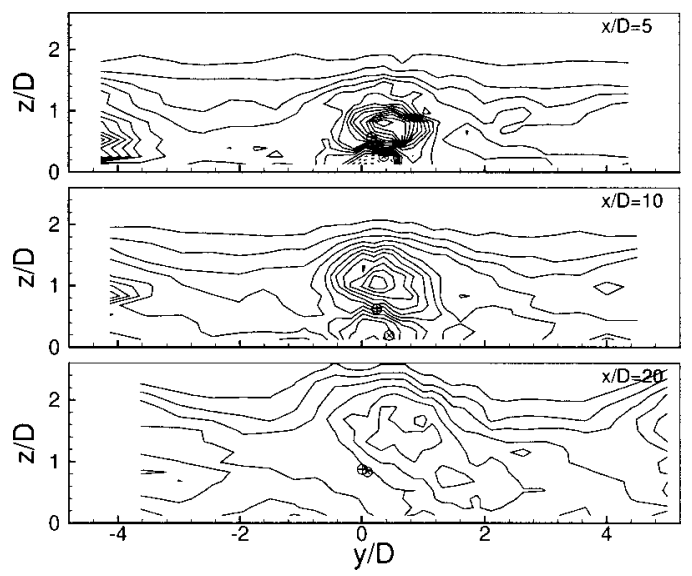

FIG. 13. Vortex development at $\lambda=0.5$; shear stress $-\overline{u^{\prime} w^{\prime}} / U_{\infty}^{2} .(\oplus)$ Center of vortex and $(\otimes)$ maximum vorticity. Contour interval 0.0003. Dashed negative lines.

the vortices evolve downstream. The high level distribution at the center of vortex is gradually reduced; the distribution becomes much smoother. At $x / D \geqslant 20$, areas of high $\overline{u^{\prime} u^{\prime}}$ distribution begin to appear on either side of the vortex close to the wall. The closed contour lines are not found at $x / D$ $=40$. Based on the normal stress development, it seems that one could define $x / D=20$ as the distance where the streamwise vortex becomes the dominant physical feature. Mean flow results reported in Secs. III B and III C have also suggested a distance between $x / D=10$ and 20 .

For the primary shear stress, $-\overline{u^{\prime} w^{\prime}}$, a distinct feature is the existence of a concentration of negative value beneath the center of vortex at $\lambda=1.5$ (Fig. 14). This feature is simi-
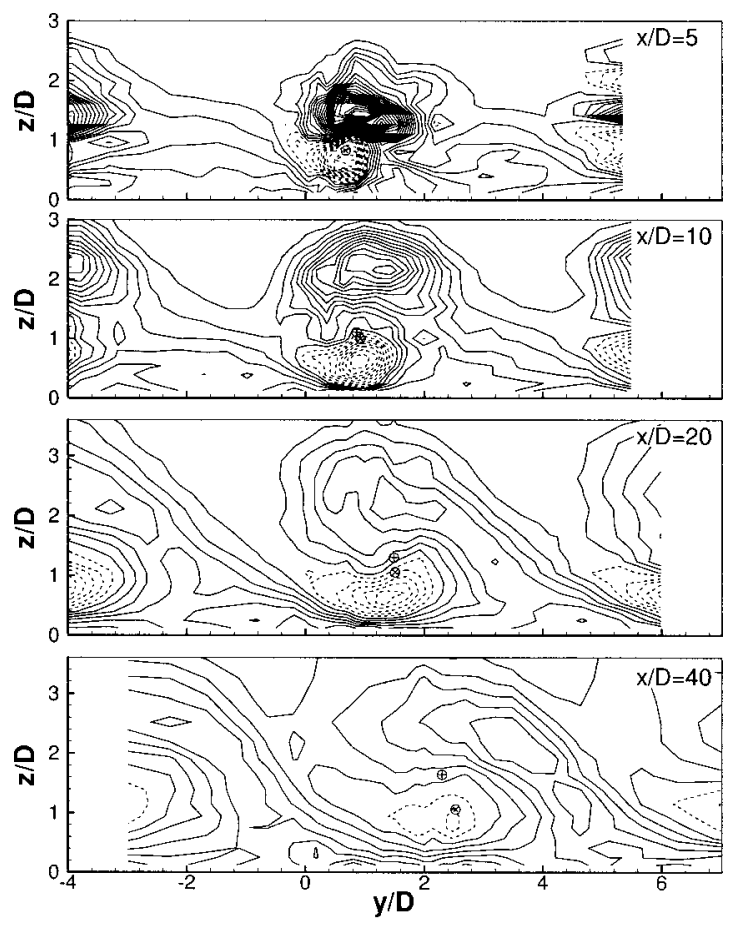

FIG. 14. Vortex development at $\lambda=1.5$; shear stress $-\overline{u^{\prime} w^{\prime}} / U_{\infty}^{2} .(\oplus)$ Center of vortex and $(\otimes)$ maximum vorticity. Contour interval 0.0003. Dashed negative lines.
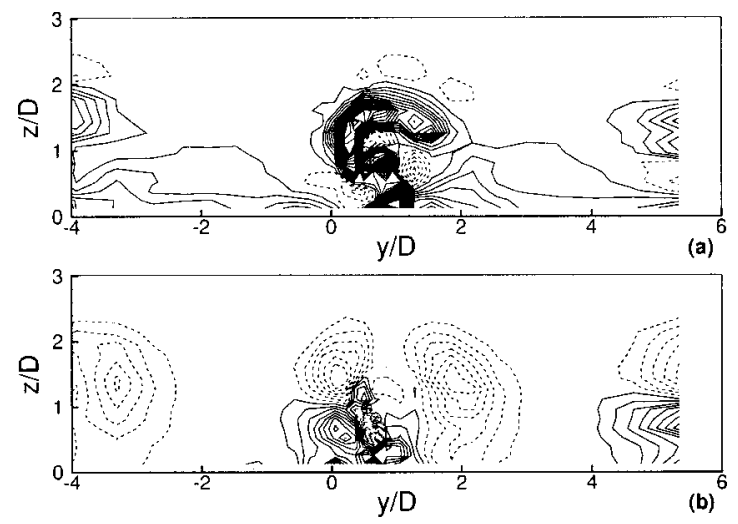

FIG. 15. The turbulence production (a) and convection (b) of normal stress $\overline{u^{\prime} u^{\prime}}$ at $x / D=5, \lambda=1.5$. Contour interval 0.0004. Dashed negative lines.

lar to that due a single jet. The concentration is marked by closed contour lines. The negative concentration is seen at all the survey positions to $x / D=40$. The negative area is separated from a concentration of high $-\overline{u^{\prime} w^{\prime}}$ by the center of vortex. The high level concentration of $-\overline{u^{\prime} w^{\prime}}$ is also marked by closed contour lines and reside above the center of vortex. The negative distribution lies between the wall and the center of vortex. However, it does not extend to the wall. Very close to the wall, the value of $-\overline{u^{\prime} w^{\prime}}$ is positive. It is clear that the primary shear stress experiences large variation across the boundary layer. In fact if one were to draw a vertical line across the boundary layer and pass the center of vortex, the value of $-\overline{u^{\prime} w^{\prime}}$ would vary in a high-low-highlow fashion. The absolute value of the lowest negative $-\overline{u^{\prime} w^{\prime}}$ is of the same order as the positive one; $0.0041 \mathrm{vs}$ 0.0067 at $x / D=5 ; 0.0024$ vs 0.0038 at $x / D=10 ; 0.0016$ vs 0.0020 at $x / D=20$. However, at $x / D=40$, the value is about three times lower than the positive one $(0.0006$ vs 0.0022$)$. At $\lambda=0.5$, there is still a region of negative $-\overline{u^{\prime} w^{\prime}}$ below the center of vortex at $x / D=5$ (Fig. 13). This negative area is not found at $x / D=10$. The influence of the jet is clearly seen by the high level concentration of $-\overline{u^{\prime} w^{\prime}}$ immediately above the center of vortex at $x / D=5$ and 10 . There are similarities between the $\lambda=0.5$ and 1.5 results.

To provide additional insight into the flow physics, we examine the turbulence production and convection of the stresses and concentrated on the normal stress, $\overline{u^{\prime} u^{\prime}}$ and the primary shear stress, $-\overline{u^{\prime} w^{\prime}}$. The $\lambda=1.5$ case is used as an example as we believe the observation will apply to other cases. The turbulence production of stress $\tau_{i j}$ is given in the Cartesian tensor notation as $-\tau_{i k} \partial \bar{v}_{j} / \partial x_{k}-\tau_{j k} \partial \bar{v}_{i} / \partial x_{k}$ and the convection term is given as $\bar{v}_{k} \partial \tau_{i j} / \partial x_{k} \cdot{ }^{10}$ In calculating these terms we assumed a "slender flow" and omitted the contribution due to the streamwise flow variation. This is believed to be reasonable based on the flow field observations, e.g., oil flow. The results for the normal stress are given in Figs. 15 and 16. Figures 17 and 18 show the results for the primary shear stress.

The turbulence production of $\overline{u^{\prime} u^{\prime}}$ in the near field shows the dominance of the jet [Fig. 15(a)]. An area of high production is found above the center of vortex. The shape 

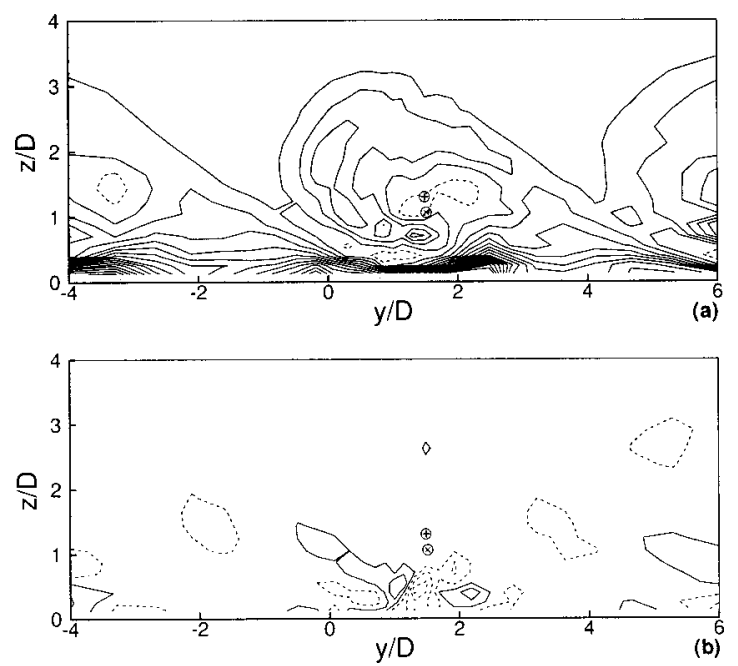

FIG. 16. The turbulence production (a) and convection (b) of normal stress $\overline{u^{\prime} u^{\prime}}$ at $x / D=20, \lambda=1.5$. Contour interval 0.0002. Dashed negative lines.

and the location of this high production value suggest that it is caused by the jet whose core is by now deformed into a tilted kidney shape. The maximum value of turbulence production in this area is 0.0078 . The turbulence convection in the near field is characterized by distinct areas of activity around the center of vortex [Fig. 15(b)]. Located above the center of vortex, there are two large areas of negative convection, to either side of the center. The minimum value in these areas is about -0.0025 . It is clear that the turbulence convection will help to shape the area of the high turbulence production above the center of vortex and will eventually reduce the area. Below the center of vortex, there are two areas of positive turbulence convection, separated by an area of negative convection. The maximum value in these positive convection areas is 0.0035 , which is of the same order in magnitude as that of the negative turbulence convection and production above the center of vortex.

At $x / D=20$ (Fig. 16), the turbulence production now shows the predominant influence of the streamwise vortex. Figure 16(a) indicates there is an area of negative production
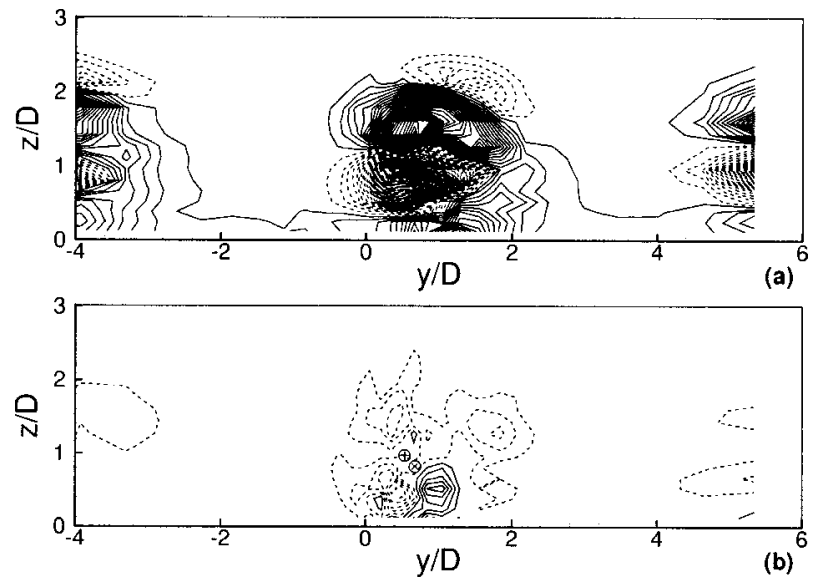

FIG. 17. The turbulence production (a) and convection (b) of primary shear stress $-\overline{u^{\prime} w^{\prime}}$ at $x / D=5, \lambda=1.5$. Contour interval 0.0002. Dashed negative lines.
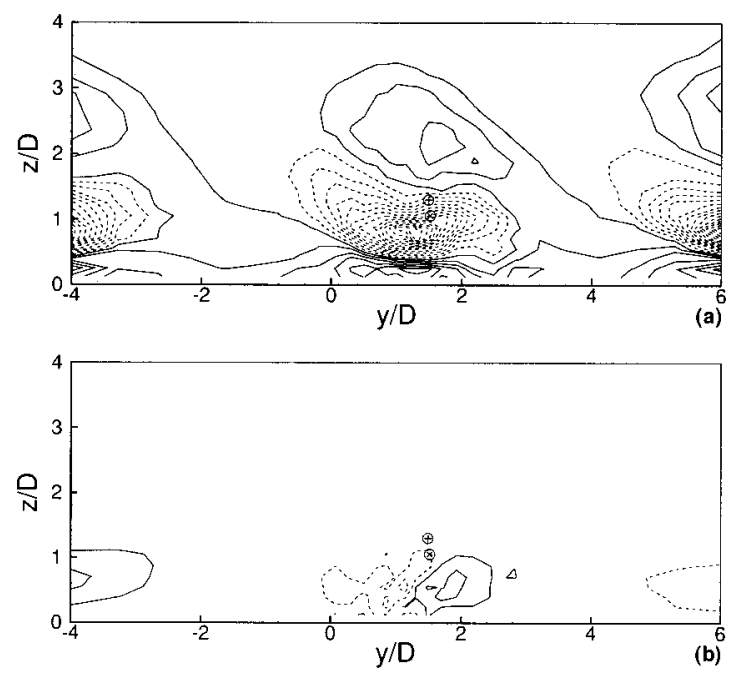

FIG. 18. The turbulence production (a) and convection (b) of primary shear stress $-\overline{u^{\prime} w^{\prime}}$ at $x / D=20, \lambda=1.5$. Contour interval 0.0002. Dashed negative lines.

near the center of vortex, which helps to smooth the $\overline{u^{\prime} u^{\prime}}$ variation and reduces its value as the vortex evolves downstream. An area of high production now appears near the wall and to the upwash side of the vortex. Turbulence convection [Fig. 16(b)] also features an area of positive value near the wall at roughly the same location. This helps to explain the high level normal stress concentration near the wall shown in Fig. 12. The turbulence convection contours now indicate major activities between the center of vortex and the wall. There are still two positive areas of convection separated by a negative area, similar to those at $x / D=5$. The maximum value in these positive areas is 0.00048 . This compares with 0.00078 found just below the center of vortex for the production term. The two areas of negative turbulence convection found in the jet near field have now disappeared.

For the primary shear stress, the dominant feature is the local turbulence production [Figs. 17(a) and 18(a)]. At both the jet near field (e.g., $x / D=5$ ) and the far field (e.g., $x / D$ $=20$ ), the basic features of the turbulence production remain the same. There is an area of negative turbulence production characterized by closed contour lines which lie mainly below the center of vortex. Unlike the primary shear stress distribution where the center of vortex separates the negative and positive areas (Fig. 14), the negative shear stress production area also covers the center of vortex. On top of the negative area there is an area of positive turbulence production also characterized by closed contour lines. Beneath the negative area, positive shear stress production is found near the wall.

At $x / D=5$, the maximum value of turbulence production is 0.0112 ; the minimum value of turbulence production is -0.027 . These values are an order of magnitude larger than those found in the turbulence convection of shear stress [Fig. 17(b)]. The turbulence convection is characterized by four distinct areas distributed around the center of vortex. Above the center of vortex there are two negative areas. Beneath the center of vortex there is one area of negative convection to the downwash side of the vortex and an area of positive convection on the upwash side of the vortex. The 
maximum value of turbulence convection is 0.0021 and the minimum value is -0.0019 . It is clear there is an order of magnitude difference between the values of production and convection.

At $x / D=20$, the basic features of the turbulence production of the shear stress are retained [Fig. 18(a)]. The maximum value of turbulence production in the area above the center of vortex is now 0.0006 ; the minimum value of turbulence production in the area below the center of vortex is now -0.0027 . These values are substantially lower than those in the near field. Near the wall, the maximum value of the positive turbulence production of the shear stress is 0.00138 . The turbulence convection of the shear stress [Fig. 18(b)] shows that of the four distinct areas observed in the near field only the two areas below the center of vortex remain. The maximum positive convection of the shear stress is 0.00035 , also an order of magnitude lower than the jet near field value. It is clear that the turbulence convection of the shear layer plays a secondary role in determining the primary shear stress distribution.

\section{SUMMARY}

The flow field dominated by co-rotating streamwise vortices and inclined jets is examined. The vortices are embedded inside a canonical flat plate boundary layer and are a result of interaction between the jets and the cross flow. The streamwise vortices are a product of complex fluid flow process, featuring horseshoe vortex in front of the nozzle exit, recirculating flow to the lee side of the nozzle, contrarotating vortices from the rolling up of vortex sheet around the jet, strong and induced spanwise flow.

The discrete streamwise vortices retain the main characters of a single streamwise vortex generated by an inclined jet. It does experience large spanwise movement.

At $\lambda=0.5$ weak vortices are produced which are located close to the wall. The vortices feature diametrically opposed secondary, near-wall flows in between the adjacent vortices. The spanwise position and induced velocity do not change significantly downstream. At $\lambda=1.0$ and 1.5 , strong vortices are produced which move in both spanwise and transverse directions. The induced secondary, near-wall flows in between the adjacent vortices are of the same direction.

As the vortices evolve downstream, their strength reduces and sizes increase. The vortices are accompanied by high level of turbulence. Distinct distributions of normal and shear stresses are found. The normal stress distributions are characterized by concentric contour lines on the cross planes in the jet near field and high levels of near wall distribution in the far field. The streamwise vortices become the dominant flow feature in the far field. Both turbulence production and convection play important roles in defining the normal stress distribution. The effect of the turbulence convection is concentrated between the wall and the center of vortex in the far field.

The primary shear stress experiences large variations in the transverse direction and is characterized by two distinct areas of positive stress (above the center of vortex) and negative (or low) stress (below the center of vortex). Unlike the normal stress, turbulence convection only plays a secondary role in defining the primary shear stress distribution. Turbulence production is the main physical mechanism and remains so in both the near and far fields.

\section{ACKNOWLEDGMENTS}

The study is supported by a U.K. EPSRC (Engineering and Physical Sciences Research Council) grant. The author would like to thank Professor M. Goodyer, of Southampton University for helpful suggestions and discussions during the course of the study.

${ }^{1} \mathrm{R}$. Wallis, “A preliminary note on a modified type of air-jet for boundarylayer control," Cp-513, Aeronautical Research Council, London, May, 1956.

${ }^{2} \mathrm{M}$. Freestone, "Preliminary tests at low speeds on the vorticity produced by air-jet vortex generators," Research Memo. Aero. 85/01, Department of Aeronautics, City University, London, February, 1985.

${ }^{3}$ J. Johnston and M. Nishi, "Vortex generator jets-a means for flow separation control," AIAA J. 28, 989 (1990).

${ }^{4}$ D. Compton and J. Johnston, "Streamwise vortex production by pitched and skewed jets in a turbulent boundary layer," AIAA J. 30, 640 (1991).

${ }^{5}$ G. Selby, J. Lin, and F. Howard, "Control of low-speed turbulent separated flow using jet vortex generators," Exp. Fluids 12, 394 (1992).

${ }^{6} \mathrm{~K}$. McManus, A. Ducharme, C. Godley, and J. Magill, "Pulsed jet actuators for suppression flow separation," in 34th AIAA Aerospace Sciences Meeting and Exhibit, Reno, NV, 15-18 January 1996, AIAA Pap. 96-0442 (1996).

${ }^{7} \mathrm{X}$. Zhang, "An inclined rectangular jet in a turbulent boundary layervortex flow," Exp. Fluids 28, 344 (2000).

${ }^{8} \mathrm{X}$. Zhang, "Turbulence measurements of a longitudinal vortex generated by an inclined jet in a turbulent boundary layer," J. Fluids Eng. 120, 765 (1998).

${ }^{9} \mathrm{X}$. Zhang, "Contra-rotating vortices embedded in a turbulent boundary layer with inclined jets," AIAA J. 37, 1277 (1999).

${ }^{10} \mathrm{~W}$. Pauly and J. Eaton, "Experiment study of the development of longitudinal vortex pairs embedded in a turbulent boundary layer," AIAA J. 26, 816 (1988).

${ }^{11} \mathrm{X}$. Zhang, "Co- and contra-rotating streamwise vortices in a turbulent boundary layer," J. Aircr. 32, 1095 (1995).

${ }^{12} \mathrm{X}$. Zhang and M. Collins, "Nearfield evolution of a longitudinal vortex generated by an inclined jet in a turbulent boundary layer,' J. Fluids Eng. 119, 934 (1997).

${ }^{13}$ R. Moffat, "Describing the uncertainties in experimental results," Exp. Fluids 1, 3 (1988).

${ }^{14}$ L. Benedict and R. Gould, "Towards better uncertainty estimates for turbulence statistics," Exp. Fluids 22, 129 (1996).

${ }^{15} \mathrm{C}$. Chen, "Aufrollung eines zylindrischen strahles durch querwind," Ph.D. thesis, University of Gottingen, Gottingen, Germany, 1942.

${ }^{16} \mathrm{D}$. Ing, "A study of the mean flow structure of circular and rectangular cxit turbulent jets initially at a small incidence angle to a uniform mainstream," Ph.D. thesis, University of Southampton, Southampton, UK, 1981

${ }^{17}$ A. Cutler and P. Bradshaw, "Strong vortex/boundary layer interactions. II. Vortices low,' Exp. Fluids 14, 393 (1993). 